SLAC-TN-10-076

LCLS-TN-05-22

\title{
Undulator Field Integral Measurements
}

\author{
Zachary Wolf \\ SLAC
}

August 5, 2005

\begin{abstract}
The LCLS undulator field integrals must be very small so that the beam trajectory slope and offset stay within tolerance. In order to make accurate measurements of the small field integrals, a long coil will be used. This note describes the design of the coil measurement system.
\end{abstract}

\section{Introduction ${ }^{1}$}

The primary tuning of the LCLS undulators will be done by sampling the magnetic field at many points and numerically calculating quantities of interest. The sampling will be done with Hall probes. For some measurements, however, the errors associated with this technique are too large. An example is the calculation of the field integrals. A small DC offset from the Hall probe, when integrated over the undulator length, can exceed the required field integral tolerances. A better technique to measure field integrals involves the use of a long coil. As the coil is moved through the field, the induced voltage is integrated to give the flux change. The field integrals can be calculated from the measured flux changes. Small offsets are easily dealt with by measuring in both the forward and backward directions. In this note, the coil measurement system under construction is described.

The maximum values for the field integrals have been specified ${ }^{2}$. The first integral of both $B_{x}$ and $B_{y}$ must be less than $40 \times 10^{-6} \mathrm{Tm}$. The second integral of both $B_{x}$ and $B_{y}$ must be less than $50 \times 10^{-6} \mathrm{Tm}^{2}$. The exact definitions of these integrals and a discussion of their importance will be given below.

The field integrals should be measured better than the required maximum values for the undulators. This is very difficult, if not impossible, to do even with the best Hall probes. Consider a Sentron Hall probe as used at ANL and many other laboratories for precision undulator measurements. The specified DC offset drift of the Sentron probe is $0.1 \mathrm{G}$, or $10^{-5} \mathrm{~T}$ (average output noise in the bandwidth $0.01 \mathrm{~Hz}$ to $100 \mathrm{~Hz}^{3}$ ). Over the $3.4 \mathrm{~m}$ length of the undulator, a $10^{-5} \mathrm{~T}$ offset would contribute $3.4 \times 10^{-5} \mathrm{Tm}$ to the first field integral, which is roughly the specified limit. Because the required Hall probe offset is at the limit of what can be achieved, a second method of measuring the field integrals is desired.

Field integral measurements are fairly easy to do with a long integrating coil, and offsets can be easily dealt with by measuring as the coil moves both forward and back, then subtracting and dividing by 2 . An additional benefit is that the equipment is somewhat mobile, unlike the granite bench required for Hall probe measurements, allowing measurements to also be done in the tunnel. Coil measurements similar to those required for the field integrals are done routinely in the magnetic

\footnotetext{
${ }^{1}$ Work supported in part by the DOE Contract DE-AC02-76SF00515. This work was performed in support of the LCLS project at SLAC.

${ }^{2}$ H. D. Nuhn et al, "LCLS Undulator Requirements", LCLS Specification \# 1.4-001 rev 2.

${ }^{3}$ Sentron model 3M12-2-2-0.2T data sheet.
} 
measurements lab, but typically with larger signals. Extra care must be taken in the present system design to make these low level measurements.

We begin the note with a discussion of the field integrals and their importance. We then discuss how to measure the field integrals with a coil. The measurement system is described. Estimates are made of the signal levels and noise. The required number of turns on the coil is determined. A second coil system for use in the tunnel is discussed.

\section{Discussion Of The Field Integrals}

The field integrals determine the overall effect of the undulator on the electron beam. Let $x$ be the horizontal position of an electron, $y$ the vertical position, and $z$ the position along the undulator. The equations of motion for the electron can be expressed as follows ${ }^{4}$

$$
\begin{aligned}
x^{\prime \prime} & =-\frac{q}{\gamma m v_{z}} B_{y} \\
y^{\prime \prime} & =\frac{q}{\gamma m v_{z}} B_{x}
\end{aligned}
$$

The prime indicates a derivative with respect to $z$. In these equations, $q$ is the electron charge, $\gamma$ is the Lorentz factor, $m$ is the electron rest mass, $v_{z}$ is the velocity along the undulator, and $B_{x}$ and $B_{y}$ are the horizontal and vertical magnetic field components, respectively.

The horizontal and vertical slopes of the trajectories are found by integrating these equations along $z$. At the initial point $z=z_{0}$, we take the initial slopes $x^{\prime}\left(z_{0}\right)$ and $y^{\prime}\left(z_{0}\right)$ to be zero. The slopes for arbitrary $z$ are given as follows.

$$
\begin{aligned}
& x^{\prime}(z)=\int_{z_{0}}^{z} x^{\prime \prime}\left(z_{1}\right) d z_{1}=-\frac{q}{\gamma m v_{z}} \int_{z_{0}}^{z} B_{y}\left(z_{1}\right) d z_{1} \\
& y^{\prime}(z)=\int_{z_{0}}^{z} y^{\prime \prime}\left(z_{1}\right) d z_{1}=\frac{q}{\gamma m v_{z}} \int_{z_{0}}^{z} B_{x}\left(z_{1}\right) d z_{1}
\end{aligned}
$$

Note that the slope depends on the first integral of the magnetic field. The exit slope from the undulator is found by integrating through the undulator. If the region of interest, which includes the undulator, has length $L$, the exit slope is given by

$$
\begin{aligned}
x_{\text {exit }}^{\prime} & =\int_{z_{0}}^{z_{0}+L} x^{\prime \prime}\left(z_{1}\right) d z_{1}=-\frac{q}{\gamma m v_{z}} \int_{z_{0}}^{z_{0}+L} B_{y}\left(z_{1}\right) d z_{1} \\
y_{\text {exit }}^{\prime} & =\int_{z_{0}}^{z_{0}+L} y^{\prime \prime}\left(z_{1}\right) d z_{1}=\frac{q}{\gamma m v_{z}} \int_{z_{0}}^{z_{0}+L} B_{x}\left(z_{1}\right) d z_{1}
\end{aligned}
$$

These equations explain why the first field integrals should be small, so that the electrons receive a minimal transverse kick as they go through the undulator.

To find the horizontal and vertical positions of the electrons, the slopes are integrated again. The initial positions $x\left(z_{0}\right)$ and $y\left(z_{0}\right)$ are taken to be zero.

$$
\begin{aligned}
& x(z)=\int_{z_{0}}^{z} x^{\prime}\left(z_{2}\right) d z_{2}=-\frac{q}{\gamma m v_{z}} \int_{z_{0}}^{z} \int_{z_{0}}^{z_{2}} B_{y}\left(z_{1}\right) d z_{1} d z_{2} \\
& y(z)=\int_{z_{0}}^{z} y^{\prime}\left(z_{2}\right) d z_{2}=\frac{q}{\gamma m v_{z}} \int_{z_{0}}^{z} \int_{z_{0}}^{z_{2}} B_{x}\left(z_{1}\right) d z_{1} d z_{2}
\end{aligned}
$$

\footnotetext{
${ }^{4}$ Z. Wolf, "Introduction To LCLS Undulator Tuning", LCLS-TN-04-7, June 2004.
} 
The positions at the exit of the undulator are given by

$$
\begin{aligned}
& x_{\text {exit }}=\int_{z_{0}}^{z_{0}+L} x^{\prime}\left(z_{2}\right) d z_{2}=-\frac{q}{\gamma m v_{z}} \int_{z_{0}}^{z_{0}+L} \int_{z_{0}}^{z_{2}} B_{y}\left(z_{1}\right) d z_{1} d z_{2} \\
& y_{\text {exit }}=\int_{z_{0}}^{z_{0}+L} y^{\prime}\left(z_{2}\right) d z_{2}=\frac{q}{\gamma m v_{z}} \int_{z_{0}}^{z_{0}+L} \int_{z_{0}}^{z_{2}} B_{x}\left(z_{1}\right) d z_{1} d z_{2}
\end{aligned}
$$

Note that in order for the electrons to receive a small transverse displacement in the undulator, the second integral of the magnetic field must be small.

We now explicitly define the field integrals as follows

$$
\begin{aligned}
I_{1 x} & \equiv \int_{z_{0}}^{z_{0}+L} B_{x}\left(z_{1}\right) d z_{1} \\
I_{1 y} & \equiv \int_{z_{0}}^{z_{0}+L} B_{y}\left(z_{1}\right) d z_{1} \\
I_{2 x} & \equiv \int_{z_{0}}^{z_{0}+L} \int_{z_{0}}^{z_{2}} B_{x}\left(z_{1}\right) d z_{1} d z_{2} \\
I_{2 y} & \equiv \int_{z_{0}}^{z_{0}+L} \int_{z_{0}}^{z_{2}} B_{y}\left(z_{1}\right) d z_{1} d z_{2}
\end{aligned}
$$

Using these definitions, we have

$$
\begin{aligned}
x_{\text {exit }}^{\prime} & =-\frac{q}{\gamma m v_{z}} I_{1 y} \\
y_{\text {exit }}^{\prime} & =\frac{q}{\gamma m v_{z}} I_{1 x} \\
x_{\text {exit }} & =-\frac{q}{\gamma m v_{z}} I_{2 y} \\
y_{\text {exit }} & =\frac{q}{\gamma m v_{z}} I_{2 x}
\end{aligned}
$$

At this point, it is interesting to put numerical values into these expressions. Approximating $v_{z}$ by $c$ and using the LCLS value of $\gamma=26693^{5}$, the factor $\frac{q}{\gamma m c}$ in these expressions has a value of $\frac{q}{\gamma m c}=2.20 \times 10^{-2} \frac{1}{T m}$. With the limits given in the introduction (for both the $x$ and $y$ directions)

$$
\begin{aligned}
& I_{1 x, y}<40 \times 10^{-6} \mathrm{Tm} \\
& I_{2 x, y}<50 \times 10^{-6} \mathrm{Tm}^{2}
\end{aligned}
$$

we find

$$
\begin{aligned}
& x_{\text {exit,max }}^{\prime}=y_{\text {exit, } \text { max }}^{\prime}=8.8 \times 10^{-7} \\
& x_{\text {exit,max }}=y_{\text {exit, } \text { max }}=1.1 \times 10^{-6} \mathrm{~m}
\end{aligned}
$$

These are obviously very tight requirements.

The form of the second field integral is suitable for sampling the field with a Hall probe and numerically integrating. It is not clear, however, how to use a coil to determine the second field integral expressed in this form. In order to have a more useful expression, we integrate by parts. Temporarily suppressing the $x$ or $y$ subscript, we have

$$
I_{2}=\int_{z_{0}}^{z_{0}+L} \int_{z_{0}}^{z_{2}} B\left(z_{1}\right) d z_{1} d z_{2}
$$

\footnotetext{
${ }^{5}$ LCLS parameter database http://www-ssrl.slac.stanford.edu/htbin/rdbweb/LCLS_params_DB_public.
} 
Let $u\left(z_{2}\right)=\int_{z_{0}}^{z_{2}} B\left(z_{1}\right) d z_{1}, d v=d z_{2}$. Then $d u=B\left(z_{2}\right) d z_{2}, v=z_{2}$. Integrating by parts, we find

$$
I_{2}=\left.z_{2} \int_{z_{0}}^{z_{2}} B\left(z_{1}\right) d z_{1}\right|_{z_{0}} ^{z_{0}+L}-\int_{z_{0}}^{z_{0}+L} z_{2} B\left(z_{2}\right) d z_{2}
$$

Putting the limits into the first term gives

$$
I_{2}=\left(z_{0}+L\right) \int_{z_{0}}^{z_{0}+L} B\left(z_{1}\right) d z_{1}-\int_{z_{0}}^{z_{0}+L} z_{2} B\left(z_{2}\right) d z_{2}
$$

Adding the field components to the notation, we can write the equations for $I_{2 x, y}$ as

$$
\begin{aligned}
& I_{2 x}=\int_{z_{0}}^{z_{0}+L}\left(z_{0}+L-z_{2}\right) B_{x}\left(z_{2}\right) d z_{2} \\
& I_{2 y}=\int_{z_{0}}^{z_{0}+L}\left(z_{0}+L-z_{2}\right) B_{y}\left(z_{2}\right) d z_{2}
\end{aligned}
$$

In this form, it is clear that the second field integrals have a weighting factor equal to the distance to the end point of the integration. This linear weighting factor allows easy interpretation for doing measurements with a coil, as shown below.

\section{Coil Measurement Technique}

The field integrals can be measured using a long coil. Consider first a wire translated parallel to itself horizontally through a magnetic field as shown in figure 1. If the return path is fixed,

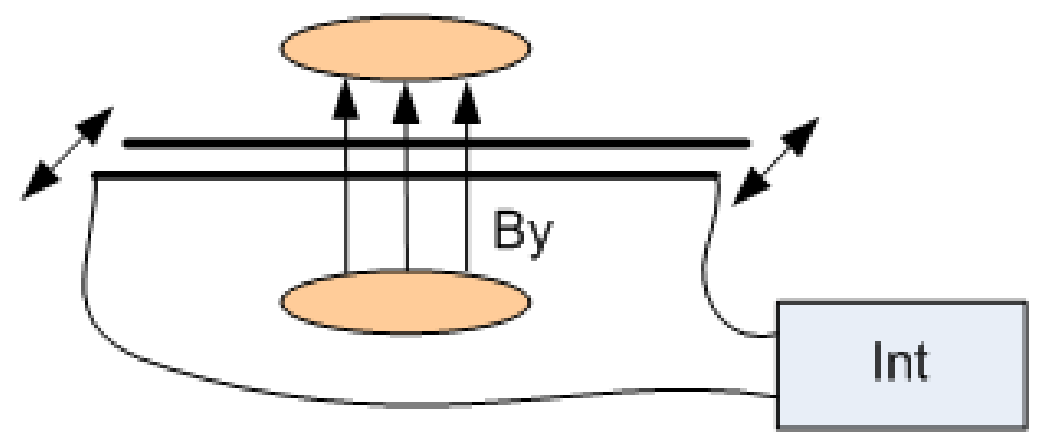

Figure 1: A wire moving through a magnetic field generates a voltage. The voltage is integrated to give the flux change.

the magnitude of the voltage induced in the circuit is the time rate of change of the magnetic flux $V=d \Phi / d t$. The voltage is sent to an integrator giving output $V T \equiv \int V d t=\int d \Phi=\Delta \Phi$. The integrator output gives the flux change in the circuit. If the wire is moved a distance $\Delta x$, the flux change is given by $\Delta \Phi=\Delta x \int B_{y} d z$. The field integral can be measured by dividing the integrator output by the distance the wire is moved.

$$
I_{1 y}=\int B_{y} d z=\frac{V T}{\Delta x}
$$

For a coil of $N$ turns, the expression becomes

$$
I_{1 y}=\int B_{y} d z=\frac{V T}{N \Delta x}
$$


Thus, the first field integral is measured by moving a bundle of wires parallel to itself through the magnetic field. Horizontal motion gives $I_{1 y}$, and vertical motion gives $I_{1 x}$.

Now suppose the exit end of the wire is held fixed and the entrance end is moved by a distance $\Delta x$. This situation is illustrated in figure 2. In this case the integrator output, which is equal to

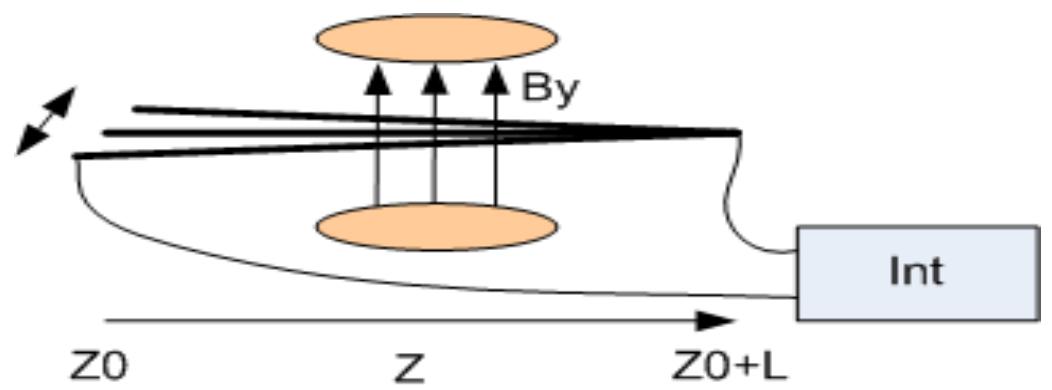

Figure 2: The undulator entrance end of the wire is moved and the exit end is held stationary to measure the second field integral.

the flux change, is given by

$$
V T=\Delta \Phi=\int_{z_{0}}^{z_{0}+L} \Delta x \frac{z_{0}+L-z}{L} B_{y} d z
$$

Thus, the second field integral is given by

$$
I_{2 y}=\int_{z_{0}}^{z_{0}+L}\left(z_{0}+L-z\right) B_{y} d z=\frac{V T L}{\Delta x}
$$

For an $N$ turn coil, this expression becomes

$$
I_{2 y}=\int_{z_{0}}^{z_{0}+L}\left(z_{0}+L-z\right) B_{y} d z=\frac{V T L}{N \Delta x}
$$

This equation illustrates that the second field integral can be easily obtained from the integrator output when one end of the wire is moved. Moving the wire horizontally gives $I_{2 y}$. Moving the wire vertically gives $I_{2 x}$.

A schematic of the coil is shown in figure 3 . The coil will have a tall vertical part to give it strength. A thin part will fit into the gap and hold the bundle of wires. The return path of the wires will be on a fixed support which does not move. The coil will be moved vertically and horizontally to make the measurement. The coil will be parked in its home position outside the undulator when not in use. The motion will be done by a set of $x y$ stages moving together at both ends of the undulator. A schematic of the overall system is shown in figure 4.

\section{System Performance}

Using the formulas from the previous section, we can calculate the expected signals from a coil measurement system and compare them to the integrator noise level. This will tell us how many turns the coil must have in order for the signal to be above the noise.

If the first field integral in the undulator is to be below the specified limit of $40 \times 10^{-6} \mathrm{Tm}$, then the measurement system must be capable of accurately measuring field integrals smaller than this, on the order of $I_{1, \text { meas }}=4 \times 10^{-6} \mathrm{Tm}$. Similarly, if the second field integrals in the undulator are 


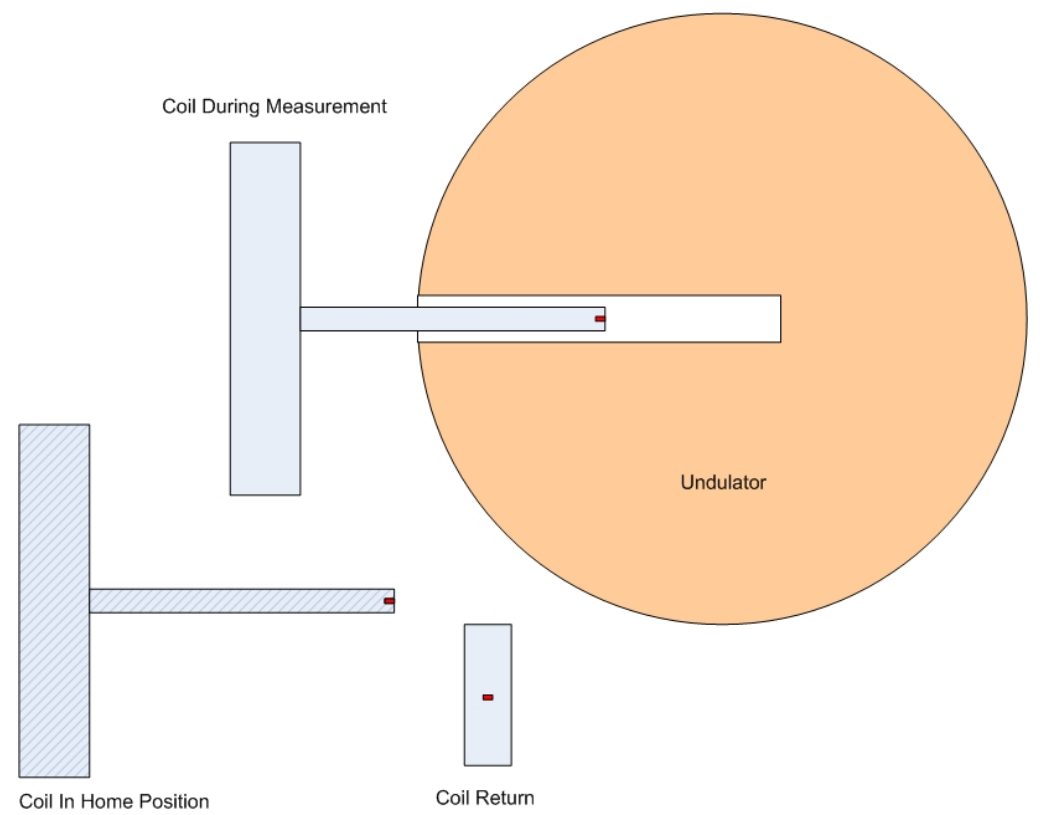

Figure 3: Schematic of the measurement coil and its return path. The coil will be parked in its home position when not in use.

to be below $50 \times 10^{-6} \mathrm{Tm}^{2}$, then the measurement system must be able to measure second field integrals on the order of $I_{2, \text { meas }}=5 \times 10^{-6} \mathrm{Tm}^{2}$. This is summarized below.

$$
\begin{aligned}
& I_{1 x, y, \text { meas }}=4 \times 10^{-6} \mathrm{Tm} \\
& I_{2 x, y, \text { meas }}=5 \times 10^{-6} \mathrm{Tm}^{2}
\end{aligned}
$$

We can calculate the signal levels corresponding to the field integral values we must measure. The LCLS undulator is $3.4 \mathrm{~m}$ long. For these calculations we take $L=3.4 \mathrm{~m}$, although the actual coil will be slightly longer. The undulator aperture is only $6.8 \mathrm{~mm}$ high. We assume that we can build a coil approximately $4 \mathrm{~mm}$ high. With appropriate clearance, we take the maximum distance that we can move the coil vertically to be $\Delta y=1 \mathrm{~mm}$. Although the coil can be moved farther in $x$, we use this $1 \mathrm{~mm}$ distance to make a worst case estimate. Using these values, we arrive at the following required measurement capabilities. From the first field integral, we get

$$
\left(\frac{V T}{N}\right)_{I_{1}}=I_{1 x, y, \text { meas }} \Delta y=\left(4 \times 10^{-6} T m\right)\left(1 \times 10^{-3} \mathrm{~m}\right)=4 \times 10^{-9} \mathrm{Tm}^{2}
$$

From the second field integral, we get

$$
\left(\frac{V T}{N}\right)_{I_{2}}=I_{2 x, y, \text { meas }} \frac{\Delta y}{L}=\left(5 \times 10^{-6} \mathrm{Tm}^{2}\right) \frac{1 \times 10^{-3} \mathrm{~m}}{3.4 \mathrm{~m}}=1.5 \times 10^{-9} \mathrm{Tm}^{2}
$$

Note that $1 \mathrm{Tm}^{2}=1 \mathrm{VS}$. Both the first and second field integral measurements lead to similar requirements on the integrated voltage measurement. We must be able to measure

$$
V T=\left(1.5 \times 10^{-9} V s\right) N
$$

where $N$ is the number of turns in the coil, as noted above. 


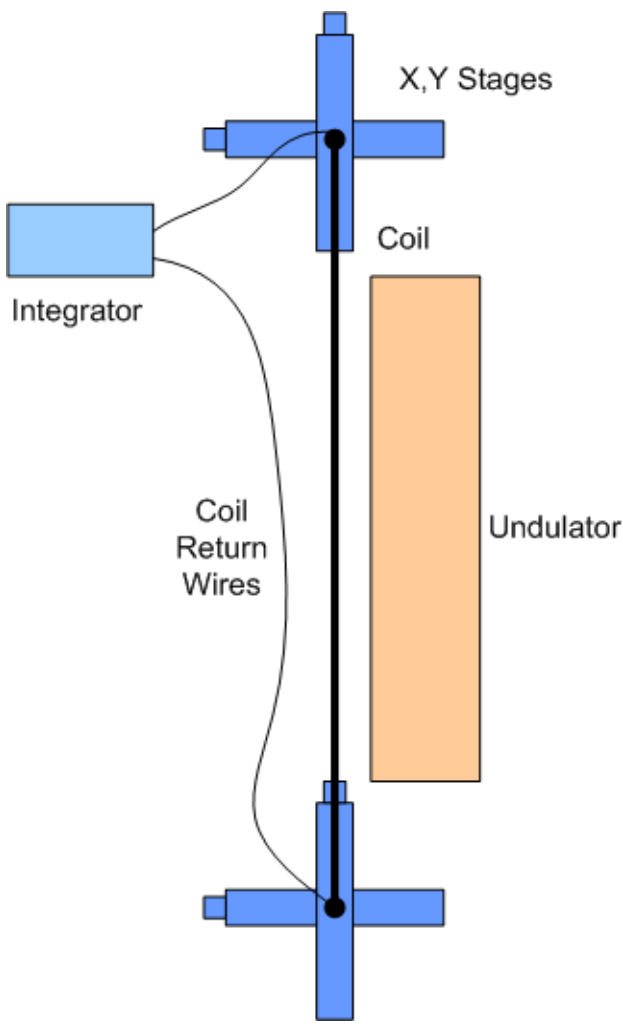

Figure 4: Coil measurement system schematic.

If the integrator is limited to signals larger than $V T_{\min }$, then we require

$$
N>\frac{V T_{\min }}{1.5 \times 10^{-9} V s}
$$

We will use a model PDI5025 digital integrator from Metrolab for these measurements. Its measurement ranges and noise performance are shown in figure 5. Suppose the coil measurement takes one second. If we use the maximum gain of 1000 , the noise level gives $V T_{\min }=2 \times 10^{-7} \mathrm{Vs}$, as indicated by the circle in the figure. Using this value for $V T_{\min }$ in the formula for the minimum number of turns on the coil gives $N>133$. Thus we need a measurement coil with at least 133 turns.

\section{Tunnel Measurements}

It has been pointed out that field integral measurements in the tunnel would be useful ${ }^{6}$. The horizontal component of the field is not expected to change between the measurement lab and the tunnel because it is caused by internal imperfections in the undulator. External horizontal fields are shielded by the magnet poles. The vertical component of the field may change, however, since it is influenced by the Earth's field and stray magnetic fields. Measuring the vertical fields is relatively easy because the coil can be moved a large distance horizontally. Suppose we move the coil $1 \mathrm{~cm}$ horizontally. The signal is 10 times larger than our previous estimate in which the coil could only

\footnotetext{
${ }^{6}$ Isaac Vasserman, private communication.
} 
The following diagram shows the measurement domains at different gains (hatched: $\mathrm{G}=10$ ) and the noise levels.

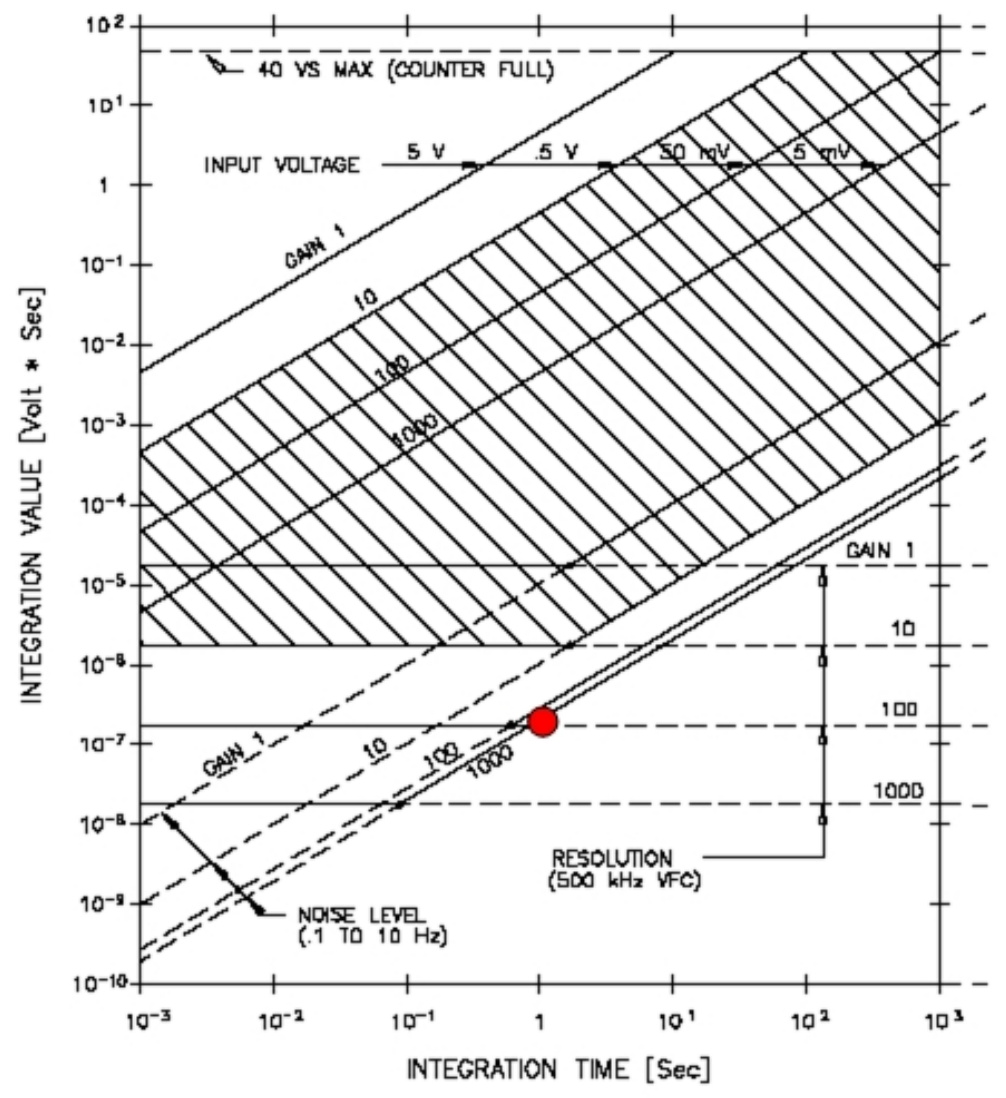

Figure 5: Plot from the PDI5052 manual showing the measurement noise levels and resolution for different gain settings.

be moved $1 \mathrm{~mm}$. Alternatively, we can use 10 times fewer turns on the coil. We plan to build a separate system for tunnel measurements which will only move the coil horizontally and will have a coil with roughly 13 turns.

The tunnel measurement system will allow us to zero the first and second field integrals near the final location of the undulator. A shim on the undulator entrance will first be adjusted to zero $I_{2 y}$. Then a shim on the undulator exit will be adjusted to zero $I_{1 y}$. These are intended to be small corrections, after the undulator has been tuned, to account for background field differences between the measurement lab and the tunnel.

\section{Conclusion}

Two systems will be built for field integral measurements. The primary system will be used in the magnetic measurement facility. It will measure the field integrals of both $B_{x}$ and $B_{y}$ with an accuracy of roughly $\Delta I_{1, x y}=4 \times 10^{-6} \mathrm{Tm}$ and $\Delta I_{2}=5 \times 10^{-6} \mathrm{Tm}^{2}$, both limited by integrator 
noise. These measurement accuracies are 10 times better than the required field integral limits on the undulators. The measurement of $B_{x}$ is made difficult because only small $(\sim 1 \mathrm{~mm})$ motions of the coil can be made due to the small size of the undulator gap. This is compensated for by having many turns on the coil $(N>133)$. Larger motions are possible for the field integrals of $B_{y}$, and correspondingly larger signals will be measured.

A second system will be used for field integral measurements in the tunnel. Only measurements of the vertical field are required. The system will only have horizontal motion. A separate coil with around 13 turns will be built. The coil will be moved roughly $1 \mathrm{~cm}$ for these measurements. The accuracy of these measurements will be the same as for the measurements in the lab. Final zeroing of the first and second field integrals of the vertical field will be done by applying shims in the tunnel.

\section{Acknowledgements}

Many thanks to Isaac Vasserman, Paul Emma, Heinz-Dieter Nuhn, and Yurii Levashov for valuable assistance with this note. 